\title{
Preeclampsia Induced Ocular Change
}

\author{
Leila Alizadeh Ghavidel ${ }^{1^{*}}$, Farideh Mousavi ${ }^{1}$, Masood Bagheri ${ }^{1}$, Saba Asghari ${ }^{1}$
}

\begin{abstract}
Objectives: Preeclampsia/eclampsia, the most common medical complication of pregnancy, can affect visual system as an end organ damage. Visual symptoms occur in $25 \%$ of patients with severe preeclampsia and $50 \%$ of patients with eclampsia. Visual system may be affected by hypertensive retinopathy, exudative retinal detachment, and cortical blindness.

Methods: A literature search was conducted using MEDLINE, EMBASE, and Cochrane library until April 2017 in English and Persian. Additionally, extensive literature was searched using the Persian databases. Moreover, the cited references in the published articles were manually reviewed for relevant results.

Results: The visual prognosis for ocular involvement was excellent in most cases because of its nature and high rate of spontaneous recovery, which occurred in a few weeks. The fact that there is a correlation between ocular finding and placental vascular abnormality emphasized correct diagnosis and evaluation of pregnant patients with visual changes. This review described the ocular involvement in pregnant patients with preeclampsia/eclampsia. The first purpose of this study was to discuss the pathophysiology and epidemiology of visual system involvement in preeclampsia/eclampsia. The second one was to diagnose the adverse effect on visual system as a last organ damage. Finally, in this review, the ophthalmologic finding, controversial issue of the treatment and prognosis with preventive care were discussed and monitoring methods for patients were followed up.

Conclusions: Visual involvement is common in pregnancy-induced hypertension and retinal vascular changes are the most common ocular findings. It seems that the progression of these changes is a sign of increasing severity of pregnancy induced hypertension (PIH) and has correlated with fetal mortality. As a result, these findings may be guideline for prompt intervention as they may reflect similar ischemic vascular changes in the placenta. Although ocular involvement has a good prognosis in PIH, repeated observations, early diagnosis, and prompt management are essential for both maternal and fetal ultimate outcomes.

Keywords: Eclampsia, Preeclampsia, Visual change
\end{abstract}

\section{Introduction}

Preeclampsia, the most common medical complication of pregnancy, is characterized by elevated systemic blood pressure ( $>140 / 100 \mathrm{~mm} \mathrm{Hg}$ ), proteinuria ( $>300$ $\mathrm{mg} / 24$ hours), and generalized edema, and it typically appears after the 20th week of pregnancy. It occurs in $5 \%-10 \%$ of all pregnancies (1). Severe preeclampsia presents with systemic blood pressure over 160/110 $\mathrm{mm} \mathrm{Hg}$, proteinuria $2 \mathrm{~g} / 24 \mathrm{~h}$, serum creatinine $>2 \mathrm{mg}$ / $\mathrm{dL}$, oliguria, thrombocytopenia, epigastric pain, cerebral and visual disruptions, headache, pulmonary edema, and elevated liver enzymes. In addition to the symptoms of preeclampsia, convulsions before or after birth indicates progression to eclampsia (1).

Preeclampsia or eclampsia may develop retinal and choroidal circulation dysfunction and various fundoscopic findings and subsequent vision loss may occur as a result. These patients may have severe hypertensive retinopathy findings such as retinal hemorrhage, subretinal serous fluid accumulation, papilledema, and Elschnig spots.

Other most common ocular complications that could be associated with (pre)eclampsia are Purtscherlike retinopathy, cortical blindness, retinal or vitreous hemorrhages serous retinal detachment, and central retinal vein occlusions. The visual symptoms may include blurred vision, double vision, sudden transient vision loss, flashing lights, and visual field complaints, including homonymous hemianopia (2). A dramatic endpoint of vision loss in preeclampsia is cortical blindness (3). This complication, though uncommon, that happens only in the severe preeclampsia is the sudden decreased vision (4). However, the loss of vision and serous retinal detachments accompanies the preeclamptic period generally and subsides in the postpartum period (5).

It is thought that the degree of changes in retina of patients with preeclampsia/eclampsia may indirectly correlate with the severity of placental vascular alterations and as a result with insufficiency of placenta and low birth weight of fetus (6). Some authors believe that all women in childbearing ages should be evaluated of preeclampsia/ eclampsia if they have any retinal or choroidal findings of malignant hypertension (7). In this review, we discuss the definitions and proposed pathophysiology of preeclampsia/eclampsia as well as its potential role in ocular morbidity. 


\section{Methods}

A literature search was conducted using MEDLINE, EMBASE, and Cochrane library until April 2017 in English and Persian with the keywords "eye", "preeclampsia", "eclampsia", and "pregnancy". Additionally, extensive literature was searched using the Persian databases (e.g. IRANMEDEX, Mediran, SID). Furthermore, the cited references in published articles were manually reviewed for relevant results.

\section{Results}

Pathophysiology

Systemic vascular involvement in preeclampsia is confirmed by the fact that there is an endothelial targeted damage in this disorder featuring with hypertension and proteinuria (8). Retinal changes seem to be the same in severe preeclampsia as in other types of systemic hypertension even without systemic changes (9).

According to a study by Karki et al, changes in the retina occur more commonly when the blood pressure is so high or proteinuria is too severe (10). In contrast to this study, Schultz and O'Brien examined the retinal and choroidal changes of 47 patients with pre-eclampsia and hypothesized that the change in retinal circulation and its severity correlates with underlying vasospasm more than the level of blood pressure (11).

Alterations in arterial wall and lumen such as narrowing are the first pathologic changes in the arterioles and their branches which occur in the mild stages of preeclampsia (12). The fact that the ratio of arteriole to vein decreases in preeclampsia suggests the retinal vasospasm and resistance to blood flow as a causative pathogenesis for visual symptoms (13). An increase in vascular permeability may also exacerbate the findings leading to extravasation of serum into the extravascular spaces. Retinal changes ensuing this extravasation may include serous retinal detachments, cotton wool spots, retinal hemorrhages, Elschnig spots, and decreased ratio of retinal arterioles to veins (6).

Sudden severe increase in blood pressure may cause choroidal ischemia leading to serous retinal detachment (14). In addition, serous retinal detachment may be caused by ischemic changes in the retinal pigment epithelium (RPE) layer. These ischemic changes destroy outer blood-retinal barrier leading to the accumulation of proteinaceous exudates in the subretinal space and the formation of serous retinal detachment (15).

Nakatsuka et al included pregnant women with and without sever preeclampsia and compared their pulsed Doppler ultrasonography's results. They found that severe preeclampsia might be associated with a decrease in the resistance of ophthalmic artery. They explained the possible direct effect on ophthalmic artery flow velocity waveform by blood flow of the internal carotid arteries and the cerebral vessels (16).

The exact pathophysiologic changes that cause cortical blindness in preeclampsia/eclampsia are not well understood however. Single-photon emission tomography (PET) has enabled some researchers to evaluate the mechanism by which cortical blindness occurs. A study performed by McCormick et al supported the finding that an increase in the permeability of capillaries may cause vasogenic edema and cortical blindness as a result (17).

Complement activation and leucocyte aggregation lead to the formation of emboli in the retinal arterioles and manifest as Purtscher retinopathy (18).

Prevalence

Although adverse events happen in $25 \%$ of all cases with pregnancy-induced hypertension, the total blindness is a rare event (19). Up to $25 \%$ of patients with severe preeclampsia and $50 \%$ of patients with eclampsia may experience visual symptoms (20). Legal blindness occurred in about $1 \%-3 \%$ of patients with eclampsia (21). The incidence of serous retinal detachment is approximately $1 \%$ in severe preeclampsia and $10 \%$ in eclampsia (22). According to Varija and collogues' study, higher prevalence of retinal change was seen in younger age of the women (23).

Saito and Tano concluded that the incidence of choroidal ischemia and its resultant transient findings (RPE lesions and serous retinal detachments) are roughly high in patients with severe preeclampsia and eclampsia. However, significant scarring was seen in only the minority of these patients (24).

Cortical blindness observed in about $1 \%-15 \%$ of patients with severe preeclampsia and eclampsia (25). Diagnosis Indocyanine green angiographic (ICG) findings in patients with preeclampsia include nonperfusion areas in the early phases of the angiogram and choroidal vasculature staining with sub retinal leakage. In the late phases of the angiogram, multiple punctate areas of blocked fluorescence may also be encountered. ICG angiographic findings indicate that the choroidal vascular changes lead to many of the retinal manifestations seen in preeclamptic patients (26).

A review of the literature on fluorescein angiography (FA) in pregnancy conducted by Schreyer et al, concluded that the primary vascular changes in preeclamptic toxemia are choroidal rather than retinal (27).

In a study by Chaine et al, ocular fluorophotometry performed in 24 patients with hypertension due to preeclampsia and in 10 normal subjects. The exclusion criteria were any fundus changes of accelerated hypertension (e.g., disc edema, cotton-wool spots, and hemorrhage). Fluorescein accumulated in the vitreous and aqueous humor of patients with preeclampsia were more significant than normal patients. This finding might indicate that the blood-aqueous barrier was damaged earlier than the blood-retinal barrier. Moreover, the damage in both barriers was a result of increased arterial 
diameter.

Fluorometric abnormalities of the eye improved after delivery in all of cases except 2 of them (28). Optical coherence tomography (OCT) showed diffuse macular edema with an increase in macular thickness that persisted after delivery but with a less significant amount. Foveal contour reversed to its normal condition 8 months after initial examination. Moreover, Elschnig spots were also visible on OCT with focal RPE abnormalities and elevated spots (29).

\section{Treatment and Prognosis}

The visual prognosis for serous retinal detachment is excellent in most cases because of its nature and high rate of spontaneous recovery which occurs in a few weeks (hastened after delivery with the absorption of fluid by RPE). Thus, the management of this condition in preeclampsia is a matter of controversy (13). Occipital lesions resulting in loss of vision might be reversed and the vision was gained in 4 hours to 8 days (30).

On the other hand, Beharier et al in a study found that preeclampsia was a significant risk factor for future ocular complications such as diabetic retinopathy and retinal detachment. There was a positive linear correlation between the severity of preeclampsia episodes and the prevalence of long term ophthalmic complications and it was important in the assessment of patients with history of complicated pregnancy (31). In the other study, preeclampsia and subsequent poor metabolic control was a potent risk factor for deterioration of retinopathy during pregnancy in type 1 diabetic patients (32).

There are some complications of rare occurrence in different studies including choroidal infarcts, papillophlebitis, retinal artery and vein occlusion, ischemic optic neuropathy, optic atrophy, optic neuritis, and thrombosis of the central retinal artery which results in permanent visual loss $(33,34)$.

In the case of permanent ocular complication in preeclampsia, the condition might be misdiagnosed as hereditary disease. These perpetual alterations are arteriolar lumen narrowing, choroidal atrophy, and retinal pigment epitheliopathy (35).

\section{Conclusions}

Visual involvement is common in pregnancy-induced hypertension. Retinal vascular changes are the most common ocular findings. Although progression of fundus changes is a sign of increasing severity of pregnancy induced hypertension (PIH), it might be independent of systemic blood pressure. In addition, it correlates with fetal mortality. As a result, visual changes may be guidelines for prompt intervention as they may reflect similar ischemic vascular changes in the placenta.

Ocular involvement has a good prognostic ability in $\mathrm{PIH}$; however, repeated observations, early diagnosis, and prompt management are essential for both maternal and fetal ultimate outcomes.

\section{Conflict of Interests}

Authors declare that they have no conflict of interests.

\section{Ethical Issues}

Not applicable.

\section{Financial Support}

None.

\section{References}

1. Perloff D. Hypertension and pregnancy-related hypertension. Cardiol Clin. 1998;16(1):79-101. doi:10.1016/ S0733-8651(05)70386-6

2. Roos NM, Wiegman MJ, Jansonius NM, Zeeman GG. Visual disturbances in (pre)eclampsia. Obstet Gynecol Surv. 2012;67(4):242-250. doi:10.1097/OGX.0b013e318250a457

3. Gregory DG, Pelak VS, Bennett JL. Diffusion-weighted magnetic resonance imaging and the evaluation of cortical blindness in preeclampsia. Surv Ophthalmol. 2003;48(6):647-650.

4. Mourelo M, Alvarez M, Diaz JL, Garcia T, Galeiras R, Freire D. Postpartum amaurosis in a woman with severe preeclampsia. Indian J Crit Care Med. 2011;15(4):227-229. doi:10.4103/0972-5229.92077

5. Dornan KJ, Mallek DR, Wittmann BK. The sequelae of serous retinal detachment in preeclampsia. Obstet Gynecol. 1982;60(5):657-663.

6. Gupta A, Kaliaperumal S, Setia S, Suchi ST, Rao VA. Retinopathy in preeclampsia: association with birth weight and uric acid level. Retina. 2008;28(8):1104-1110. doi:10.1097/IAE.0b013e3181744122

7. Araujo J, Tavares-Ferreira J, Penas S, Figueira L, Paiva FP, Falcao-Reis F. Malignant hypertensive retinopathy as a presenting sign of an occult dead fetus. Clin Ophthalmol. 2015;9:971-975. doi:10.2147/opth.s71246

8. Thadhani R, Ecker JL, Kettyle E, Sandler L, Frigoletto FD. Pulse pressure and risk of preeclampsia: a prospective study. Obstet Gynecol. 2001;97(4):515-520.

9. Oluleye ST, Olusanya BA, Adeoye AM. Retinal vascular changes in hypertensive patients in Ibadan, Sub-Saharan Africa. Int J Gen Med. 2016;9:285-290. doi:10.2147/ijgm. s107241

10. Karki P, Malla P, Das H, Uprety DK. Association between pregnancy-induced hypertensive fundus changes and fetal outcomes. Nepal J Ophthalmol. 2010;2(1):26-30.

11. Schultz JF, O'Brien CS. Retinal Changes in Hypertensive Toxemia of Pregnancy*: A Report of 47 Cases. Am J Ophthalmol. 1938;21(7):767-774. doi:10.1016/S00029394(38)93525-1

12. Hallum AV. Eye changes in hypertensive toxemia of pregnancy: A study of three hundred cases. JAMA. 1936;106(19):1649-1651. doi:10.1001/ jama.1936.02770190025009

13. Jaffe G, Schatz H. Ocular manifestations of preeclampsia. Am J Ophthalmol. 1987;103(3 Pt 1):309-315.

14. Hayreh SS, Servais GE, Virdi PS. Fundus lesions in malignant hypertension. VI. Hypertensive choroidopathy. Ophthalmology. 1986;93(11):1383-1400.

15. Tranos PG, Wickremasinghe SS, Hundal KS, Foster PJ, Jagger 
J. Bilateral serous retinal detachment as a complication of HELLP syndrome. Eye (Lond). 2002;16(4):491-492. doi:10.1038/sj.eye.6700056

16. Nakatsuka M, Takata M, Tada K, Kudo T. Effect of a nitric oxide donor on the ophthalmic artery flow velocity waveform in preeclamptic women. J Ultrasound Med. 2002;21(3):309-313.

17. McCormick AS, Wood A, Wee MY. Visual disturbances and seizures associated with pregnancy: a diagnostic dilemma and the role of radiological techniques as an aid to diagnosis. Int J Obstet Anesth. 1999;8(3):184-188.

18. Mihu D, Mihu CM, Talu S, Costin N, Ciuchina S, Malutan A. [Ocular changes in preeclampsia]. Oftalmologia. 2008;52(2):16-22.

19. Rahman J, Rahman W. Temporary blindness as a complication of eclampsia: observations on three cases. J Obstet Gynaecol. 2002;22(1):87-88.

20. Samra KA. The eye and visual system in the preeclampsia/ eclampsia syndrome: What to expect? Saudi Journal of Ophthalmology. 2013;27(1):51-3.

21. Torres PJ, Antolin E, Gratacos E, Chamorro A, Cararach V. Cortical blindness in preeclampsia: diagnostic evaluation by transcranial Doppler and magnetic resonance imaging techniques. Acta Obstet Gynecol Scand. 1995;74(8):642644.

22. Sunness J. Pregnancy and retinal disease. Retina St Louis: Mosby; 1994.

23. Varija T, Vanaja D, Sindhura, Raghavenda B. A study of prevalence and association of fundus changes in pregnancy induced hypertension. Int J Reprod Contracept Obstet Gynecol. 2016;5(5):1375-1379. doi:10.18203/2320-1770. ijrcog20161289

24. Saito Y, Tano Y. Retinal pigment epithelial lesions associated with choroidal ischemia in preeclampsia. Retina. 1998;18(2):103-108.

25. Cunningham FG, Fernandez CO, Hernandez C. Blindness associated with preeclampsia and eclampsia. Am J Obstet
Gynecol. 1995;172(4):1291-1298. doi:10.1016/00029378(95)91495-1

26. Valluri S, Adelberg DA, Curtis RS, Olk RJ. Diagnostic indocyanine green angiography in preeclampsia. Am J Ophthalmol. 1996;122(5):672-677.

27. Schreyer P, Tzadok J, Sherman DJ, Herman A, Bar-Itzhak R, Caspi E. Fluorescein angiography in hypertensive pregnancies. Int J Gynaecol Obstet. 1991;34(2):127-132.

28. Chaine G, Attali P, Gaudric A, Colin MC, Quentel G, Coscas G. Ocular fluorophotometric and angiographic findings in toxemia of pregnancy. Arch Ophthalmol. 1986;104(11):1632-1635.

29. Theodossiadis PG, Kollia AK, Gogas P, Panagiotidis D, Moschos M, Theodossiadis GP. Retinal disorders in preeclampsia studied with optical coherence tomography. Am J Ophthalmol. 2002;133(5):707-709.

30. Swende TZ, Abwa T. Reversible blindness in fulminating preeclampsia. Ann Afr Med. 2009;8(3):189-191. doi:10.4103/1596-3519.57247

31. Beharier O, Davidson E, Sergienko R, et al. Preeclampsia and Future Risk for Maternal Ophthalmic Complications. Am J Perinatol. 2016;33(7):703-707. doi:10.1055/s-0036-1571321

32. Lovestam-Adrian M, Agardh CD, Aberg A, Agardh E. Pre-eclampsia is a potent risk factor for deterioration of retinopathy during pregnancy in Type 1 diabetic patients. Diabet Med. 1997;14(12):1059-1065. doi:10.1002/ (sici)1096-9136(199712)14:12<1059::aid-dia505>3.0.co;2-8

33. Leiba H, Glaser JS, Schatz NJ, Siatkowski RM. Postpartum optic neuritis: etiologic and pathophysiologic considerations. J Neuroophthalmol. 2000;20(2):85-88.

34. Lara-Torre E, Lee MS, Wolf MA, Shah DM. Bilateral retinal occlusion progressing to long-lasting blindness in severe preeclampsia. Obstet Gynecol. 2002;100(5 Pt 1):940-942.

35. Gass DM, Pautler SE. Toxemia of pregnancy pigment epitheliopathy masquerading as a heredomacular dystrophy. Trans Am Ophthalmol Soc. 1985;83:114-130.

(C) 2018 The Author (s); This is an open-access article distributed under the terms of the Creative Commons Attribution License (http://creativecommons.org/licenses/by/4.0), which permits unrestricted use, distribution, and reproduction in any medium, provided the original work is properly cited. 